\title{
A Survey of Close, Young Stars with SDI at the VLT and MMT
}

\author{
Beth A. Biller ${ }^{1}$, Laird M. Close ${ }^{1}$, Elena Masciadri ${ }^{2}$, Rainer Lenzen ${ }^{2}$, \\ Wolfgang Brandner ${ }^{2}$, Donald McCarthy ${ }^{1}$, Thomas Henning ${ }^{2}$, \\ Eric Nielsen ${ }^{1}$, and Markus Hartung ${ }^{3}$ \\ ${ }^{1}$ Steward Observatory, University of Arizona, Tucson, AZ 85721 \\ email: bbiller@as.arizona.edu \\ ${ }^{2}$ Max-Planck-Institut für Astronomie, Königstuhl 17, 69117 Heidelberg, Germany \\ ${ }^{3}$ European Southern Observatory, Alonso de Cordova 3107, Santiago 19, Chile
}

\begin{abstract}
We discuss the preliminary results of a survey of young $(<300 \mathrm{Myr})$, close $(<50 \mathrm{pc})$ stars with the Simultaneous Differential Extrasolar Planet Imager (SDI) implemented at the VLT and the MMT. SDI uses a quad filter to take images simultaneously at 3 wavelengths surrounding the $1.62 \mu \mathrm{m}$ methane bandhead found in the spectrum of cool brown dwarfs and gas giants. By performing a difference of images in these filters, speckle noise from the primary can be significantly attenuated, resulting in photon noise limited data. In our survey data, we achieved $\mathrm{H}$ band contrasts $>25000(5 \sigma \Delta \mathrm{F} 1(1.575 \mu \mathrm{m})>10 \mathrm{mag}, \Delta \mathrm{H}>11.5 \mathrm{mag}$ for a T6 spectral type) at a separation of 0.5 " from the primary star. With this degree of attenuation, we should be able to image ( $5 \sigma$ detection) a 2-4 Jupiter mass planet at $5 \mathrm{AU}$ around a $30 \mathrm{Myr}$ star at 10 pc. We are currently completing our survey of young, nearby stars, with complete datasets for 35 stars in the southern sky (VLT) and 7 stars in the northern sky (MMT). We believe that our SDI images are the highest contrast astronomical images ever made from ground or space for methane rich companions.
\end{abstract}

Keywords. (stars:) planetary systems, instrumentation: adaptive optics.

\section{Introduction}

Direct detection of extrasolar giant planets is extremely difficult. Giant gas planets seen in reflected light are $>20$ magnitudes fainter than their primary stars and likely lie within $\sim 1$ " of their primary stars. The problem is slightly easier with younger, hotter planets - 100 Myr old extra-solar planets are $10^{4-7}$ times more self-luminous than old (5 Gyr) extra-solar planets, whereas their primary stars are only slightly (2-5 times) brighter at early ages. In theory, adaptive optics (AO) systems that are "photon noise limited" can detect an object up to $10^{5}$ times fainter than its primary at separations $>1$ ". However, numerous surveys for extrasolar planets using large telescopes with AO systems have yielded useful limits but few confirmed giant planet candidates (Kaisler et al. 2003, Masciadri et al. 2005, Chauvin et al. 2005, Neuhäuser et al. 2005).

AO surveys for young extrasolar planets only address half of the difficulty of direct detection - the contrast limit problem. Beyond the contrast limit problem, all AO systems suffer from a limiting "speckle noise" floor (Racine et al. 1999). Within 1" of the primary star, the field is filled with speckles left over from instrumental features and residual atmospheric turbulence after adaptive optics correction. These speckles vary as a function of time and color. For photon noise limited data, the signal to noise $\mathrm{S} / \mathrm{N}$ is proportional to $\mathrm{t}^{0.5}$, where $\mathrm{t}$ is the exposure time. For speckle-noise limited data, the $\mathrm{S} / \mathrm{N}$ does not increase with time past a specific speckle-noise floor (limiting contrasts to $\sim 10^{3}$ at $0.5 "$ ). 


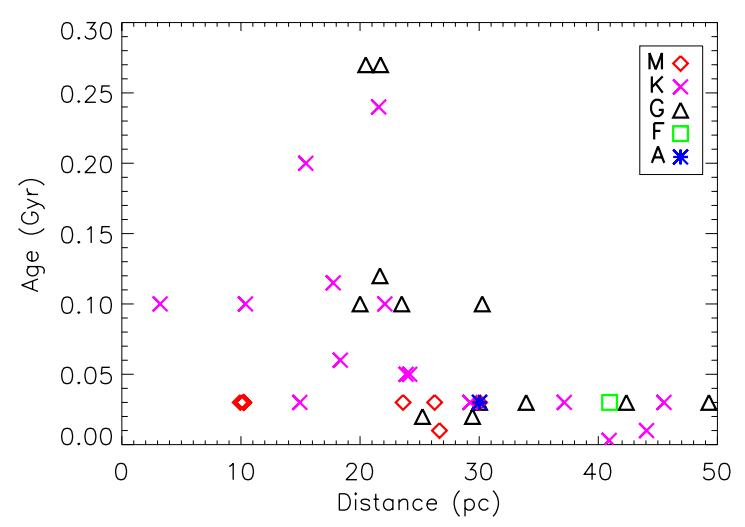

Figure 1. Age vs. Distance for our Observed Sample Stars.

This speckle-noise floor is considerably above the photon noise limit and makes planet detection very difficult. Interestingly, space telescopes such as HST also suffer from a similar limiting speckle-noise floor due to imperfect optics and "breathing" (Schneider et al. 2003). Direct detection of extrasolar giant planets requires special new instrumentation to suppress this speckle noise floor and produce photon noise limited images. The VLT, Keck, Subaru, and Gemini are all currently developing dedicated planet-finding cameras which exploit these new instrumentational approaches for speckle suppression. The Simultaneous Differential Imager (SDI), which our team built and installed at the VLT and MMT (see Biller et al., this conference), is one of the first dedicated planet-finding instruments to come online.

\section{The SDI survey}

\section{An Example Dataset}

Simultaneous Differential Imaging is an instrumental method which can be used to calibrate and remove the "speckle noise" in AO images, while also isolating the planetary light from the starlight. This method was pioneered by Racine et al. (1999), Marois et al. (2000), Marois et al. (2002), and Marois et al. (2005). It exploits the fact that all cool $\left(\mathrm{T}_{\text {eff }}<1200 \mathrm{~K}\right)$ extra-solar giant planets have strong $\mathrm{CH}_{4}$ (methane) absorption redwards of $1.62 \mu \mathrm{m}$ in the $\mathrm{H}$ band infrared atmospheric window (Burrows et al. 2001, Burrows et al. 2003). Our SDI device obtains four images of a star simultaneously through three slightly different narrowband filters (sampling both inside and outside of the $\mathrm{CH}_{4}$ features). These images are then differenced. This subtracts out the halo and speckles from the bright star to reveal any massive extrasolar planets orbiting that star. Since a massive planetary companion will be brightest in one filter and absorbed in the rest, while the star is bright in all three, a difference can be chosen which subtracts out the star's light and reveals the light from the companion. Thus, SDI also helps eliminate the large contrast difference between the star and substellar companions (Close et al. 2005; Lenzen et al. 2004; Lenzen et al. 2005) The SDI device has already produced a number of important scientific results: the discovery of AB Dor C (Close et al. 2005) which is the tightest $(0.16 ")$ low mass companion known, the first surface maps of Titan (Hartung et al. 2004), and the discovery of $\epsilon$ Indi Ba-Bb, the nearest binary brown dwarf (McCaughrean et al. 2005). 

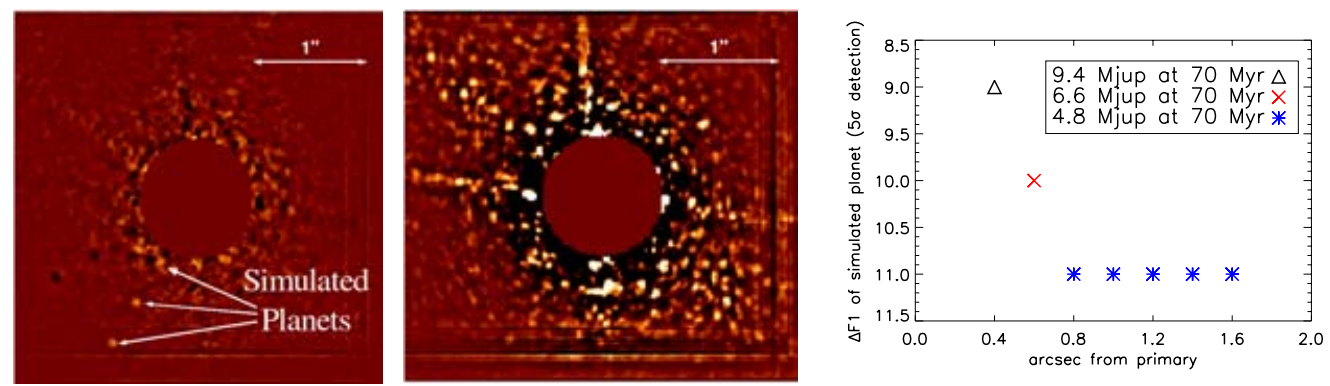

Figure 2. Left: A complete reduced dataset (40 minutes of data at a series of rotator angles $-0^{\circ}$, $33^{\circ}, 33^{\circ}, 0^{\circ}$ ) from the VLT SDI device. Simulated planets have been added at separations of 0.55 , 0.85 , and 1.35 " from the primary, with $\Delta \mathrm{F} 1(1.575 \mu \mathrm{m})=10 \mathrm{mag}$ (attenuation in magnitudes in the $1.575 \mu \mathrm{m} \mathrm{F} 1$ filter) fainter than the primary. These planets are scaled from unsaturated images of the example star taken right before the example dataset (and have fluxes and photon noise in each filter appropriate for a T6 object). Past 0.7", the simulated planets are detected with S/N > 10. Center: Standard AO data reduction of the same dataset. Filter images have been coadded (rather than subtracted), flat-fielded, sky-subtracted, and unsharp-masked. Simulated planets have been added with the same properties and at the same separations as before. None of the simulated planets are detected in the standard AO reduction. Additionally, numerous bright super speckles remain in the field. Right: Minimum Detectable Planet Mass ( $5 \sigma$ detection vs. Separation for this example dataset (Case B). To determine minimum detectable planet mass as a function of separation, we inserted and then attempted to retrieve simulated planets with a variety of separations and $\Delta \mathrm{F} 1(5 \sigma$ attenuation in magnitudes in the $1.575 \mu \mathrm{m}$ F1 filter) contrasts. $\Delta \mathrm{F} 1$ contrasts were translated into planet masses using the models of Burrows et al. 2003. For this particular star, we can detect a $5 \mathrm{M}_{J u p}$ planet $12 \mathrm{AU}$ from the star.

Table 1. Properties of Example SDI Survey Stars and Comparison Stars

\begin{tabular}{lcccccccc}
\hline Case & Spectral Type & Age & Distance & $\mathrm{H}$ & $\mathrm{V}$ & $\Delta \mathrm{F} 1^{1}$ & $\Delta \mathrm{H}^{1}$ & Exposure Time \\
\hline $\mathrm{A}$ & $\mathrm{K} 2 \mathrm{~V}$ & $30 \mathrm{Myr}$ & $45.5 \mathrm{pc}$ & 7.1 & 9.1 & 10.5 & 12 & $40 \mathrm{~min}$ \\
$\mathrm{~B}$ & $\mathrm{~K} 1 \mathrm{~V}$ & $70 \mathrm{Myr}$ & $15 \mathrm{pc}$ & 4.8 & 6.9 & 10.5 & 12 & $40 \mathrm{~min}$ \\
$\mathrm{C}$ & $\mathrm{M} 3 \mathrm{~V}$ & $30 \mathrm{Myr}$ & $24 \mathrm{pc}$ & 7.1 & 12.2 & 10 & 11.5 & $40 \mathrm{~min}$ \\
$10 \mathrm{~K}-\mathrm{M}$ stars $^{2}$ & $\mathrm{~K}-\mathrm{M}$ & $0-1 \mathrm{Gyr}$ & $10-50 \mathrm{pc}$ & $6.4-8.7$ & $8-12$ & 8.61 & 10.31 & $10-25 \mathrm{~min}$ \\
Gl $86^{3}$ & $\mathrm{~K} 1 \mathrm{~V}$ & $10 \mathrm{Gyr}$ & $10.9 \mathrm{pc}$ & 4.2 & 6.2 & 12.8 & 14.3 & $80 \mathrm{~min}$ \\
\hline
\end{tabular}

${ }^{1} 5 \sigma$ at $0.5 "{ }^{2}$ Masciadri et al. IAUC $200{ }^{3}$ Mugrauer \& Neuhäuser 2005

We are currently completing a survey with the SDI device of $\sim 50$ young $(<300 \mathrm{Myr})$, nearby $(<50 \mathrm{pc})$ stars. Stars were chosen based on strong lithium absorption features (our best targets have Li equivalent widths of $>100 \mathrm{~m} \AA$ from the $\mathrm{Li} 6707 \AA$ line, corresponding to age $<100 \mathrm{Myr}$ ) and accurate Hipparcos parallax measurements (parallaxes of $>0.02$ ", corresponding to distances $<50 \mathrm{pc}$ ). Complete datasets have been acquired for 42 stars total -35 stars in the southern sky and 7 stars in the northern sky. Ages, distances, and spectral types of observed objects are presented in Fig. 1. The "average" survey object is a late $\mathrm{K}$ star with an age of $120 \mathrm{Myr}$ and at a distance of $26 \mathrm{pc}$.

Based on realistic scaling laws for the semimajor axis, eccentricity, mass power law, and luminosity of extrasolar planets (statistics from Marcy et al. 2003 and Lineweaver and Grether 2003, masses and ages from Burrows et al. 2003) and scaling to semimajor axes $>5 \mathrm{AU}$, we can determine a rough detection probability for each program star (read Nielsen et al. this conference for more detail). Our program stars have average detection probabilities of $\sim 10-20 \%$. Integraing over the probability distribution of our program stars yields $\sim 4$ likely detections in 50 stars. 

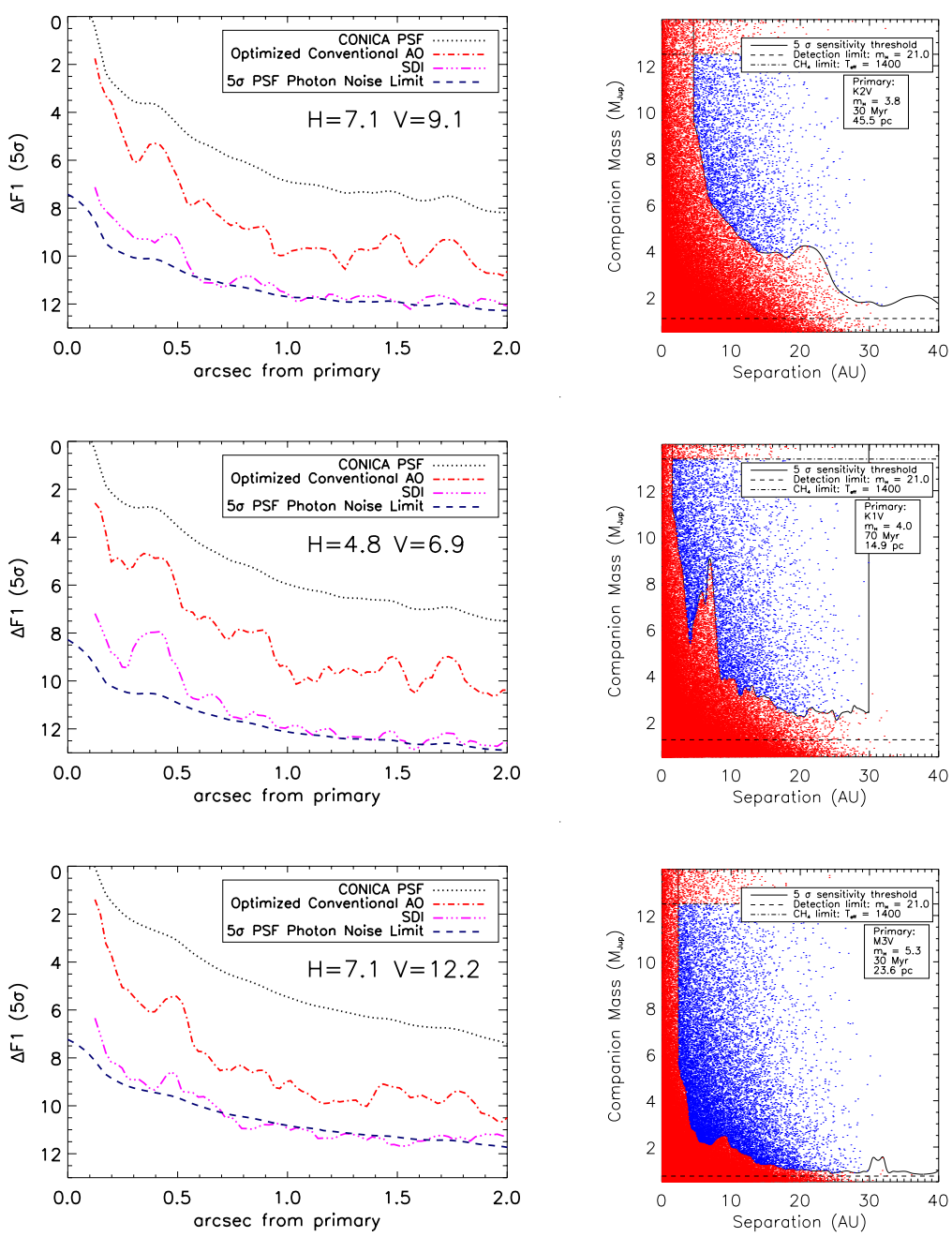

Figure 3. Top: Case A, Middle: Case B, Bottom: Case C. Left: $\Delta \mathrm{F} 1$ ( $5 \sigma$ attenuation in magnitudes in the $1.575 \mu \mathrm{m} \mathrm{F1}$ filter) vs. Separation for 40 minutes of VLT SDI data for each example star. The top curve is the AO PSF. The next curve is the "classical AO PSF" unsharp masked. The third curve down is 40 minutes of SDI data taken at two different position angles and subtracted $\left(0^{\circ}\right.$ data $-33^{\circ}$ data). The last curve is the theoretical contrast limit due to photon-noise. For each case, at star-companion separations $>0.5$ ", we are photon-noise limited and achieve $\mathrm{H}$ band star to planet contrasts $>25000(5 \sigma \Delta \mathrm{F} 1(1.575 \mu \mathrm{m})>10 \mathrm{mag}, \Delta \mathrm{H}>11.5 \mathrm{mag}$ for a T6 spectral type) Right: Minimum Detectable Planet Mass vs. Separation - using the models of Burrows et al. (2003) and primary star properties from the literature, we can convert our $\Delta \mathrm{F} 1$ values into a minimum detectable planet mass for each object. Objects above the $1400 \mathrm{~K}$ methane cutoff line (horizontal dashed line) are not detected with the SDI device. For details, see Nielsen et al. 2005 (this conference).

A number of tentative candidate extrasolar planets (objects which showed $\mathrm{CH}_{4}$ absorption at appropriate separations from the primary) have been identified so far - followup observations of these candidates are planned to confirm if these candidates share common proper motion with their parent stars. Low resolution follow-up CONICA grism spectroscopy will be performed on any confirmed candidate once the exact planet location is 
confirmed. With our excellent spatial resolution, we can determine an extremely accurate offset between the planet and thus will be able to precisely align a narrow 0.1 " slit on the planet. See Close et al. 2005 and Nielsen et al. 2005 for details on spectral reduction.

A fully reduced dataset from the VLT SDI device as well as the same dataset reduced in a standard AO manner is presented in Fig. 2. This is 40 minutes of data for AB Dor A, a 70 Myr K1V star at a distance of 14.98 pc $(\mathrm{V}=6.88)$. Simulated planets have been added at separations of $0.55,0.85$, and $1.35 "$ from the primary, with $\Delta \mathrm{F} 1=10 \mathrm{mag}$ (attenuation in magnitudes in the F1 $1.575 \mu \mathrm{m}$ filter) fainter than the primary. These planets are scaled from unsaturated images of the example star taken right before the example dataset (and have fluxes and photon noise in each filter appropriate for a T6 object). In the SDI reduction, the simulated planets are detected with $\mathrm{S} / \mathrm{N}>10$ past 0.7". In comparison, none of the simulated planets are detected in the standard AO data reduction and numerous bright super speckles remain in the field.

\section{Contrast Limits and Planet Detectability}

To determine the range of star-planet contrasts achievable in our SDI young stars survey, we consider three example cases which span the space of our target stars: case A a high quality dataset (observed with seeing of $\sim 0.5$ "), case B - AB Dor A, a young solar analogue, and case C, a faint young M star. Properties of each example star (distance, age, spectral type, etc.) are presented in Table 1.

$\Delta \mathrm{F} 1$ ( $5 \sigma$ attenuation in magnitudes in the $1.575 \mu \mathrm{m}$ F1 filter) vs. separation from the primary is presented for each of the example cases in Fig. 3. For these datasets, we achieved $\mathrm{H}$ band star to planet contrasts $>25000(5 \sigma \Delta \mathrm{F} 1(1.575 \mu \mathrm{m})>10 \mathrm{mag}, \Delta \mathrm{H}>11.5$ mag for a T6 spectral type) at a separation of 0.5 " from the primary star - approaching the photon-noise limit in 40 minutes of data.

Using the models of Burrows et al. (2003) and adopting values for the primary star's age (from the Li $6707 \AA$ line), distance, and spectral type from the literature, we can convert our measured attenuations for each object into a minimum detectable mass (see Nielsen et al. 2005, this conference). Minimum detectable mass vs. separation for each of the examples is also presented in Fig. 3. Although we achieve similar contrast limits for our example cases (with slightly higher contrasts for brighter targets as one might expect), the mass and separation of objects detectable around each varies strongly with age and distance. Even though case $\mathrm{A}$ was our best quality data, we are more likely to detect planets for case B and C, simply because these two objects are closer to the sun, and hence, we can resolve the inner $\sim 20$ AU around the star. For case $\mathrm{C}$, we can detect $(>5 \sigma)$ a $3-5 \mathrm{M}_{J}$ planet at $6 \mathrm{AU}$ from the primary. $\Delta \mathrm{F} 1$ and $\Delta \mathrm{H}$ (for a methane object) for each survey case as well as for two other comparison objects are shown in Table 1 it is clear that the achievable contrast varies according to the magnitude of the object and total exposure time.

To determine what sort of objects we can realistically detect with this level of contrast, we inserted and then attempted to retrieve simulated T6 dwarf planets to the case B dataset with a variety of separations and $\Delta \mathrm{F} 1$ contrasts. $\Delta \mathrm{F} 1$ contrasts were translated into planet masses using the models of Burrows et al. 2003. In Fig. 2, we plot minimum detectable planet mass (for a $5 \sigma$ detection) vs. separation. For this particular star (case B: K1V, $70 \mathrm{Myr}, 15 \mathrm{pc}$ ), we can detect a $5 \mathrm{M}_{\text {Jup }}$ planet $12 \mathrm{AU}$ from the star. In this particular case we were able to detect a non-methane companion (AB Dor C) at 3 different epochs and separations from 0.15 " to 0.2 " even though $\Delta \mathrm{H}>5$ mag (Close et al. this conference and Nielsen et al. 2005). 


\section{Conclusions}

The novel SDI device at the VLT and MMT has been fully commissioned and is currently achieving attenuations of $>25000(\Delta \mathrm{H}>12.5$ for a T6 spectral type object, $\Delta \mathrm{F} 1(1.575 \mu \mathrm{m})>10$ at $0.5 ")$. With these contrasts, we can detect a wide range of substellar objects. For instance, for AB Dor A (a 70 Myr K1V star 15 pc away) we can detect $(>5 \sigma)$ a $5 \mathrm{M}_{J u p}$ planet $12 \mathrm{AU}$ from the star. For a younger closer star (30 Myr age at $\left.10 \mathrm{pc}\right)$, we can detect a $2-4 \mathrm{M}_{J u p}$ planet at $5 \mathrm{AU}$.

We have currently observed 42 of the youngest $(<300 \mathrm{Myr})$, nearest $(<50 \mathrm{pc})$ stars as part of a survey of young, nearby stars. We have received time at the VLT for followup observations of 8 tentative candidates found as part of the survey. With a total sample size of $\sim 50$ stars, we will be able to place strong constraints on the frequency and semimajor axis distribution of massive extrasolar planets $>5$ AU from their primaries. From scaling laws derived from the distribution of known radial velocity planets (Marcy et al. 2003, Lineweaver and Grether 2003, Burrows et al. 2003), we expect to detect $\sim 4$ planets for our total sample (see Nielsen et al., this conference). Whether or not we detect planets, our survey will begin to measure the true distribution young massive extrasolar planets $>5 \mathrm{AU}$ from their primaries and will provide valuable contraints for theories of planet formation and migration.

\section{Acknowledgements}

BAB acknowledges support through the NASA GSRP program. LMC acknowledges support through NSF CAREER and NASA Origins grants.

\section{References}

Burrows, A., Hubbard, W.B., Lunine, J., \& Liebert, J. 2001, Reviews of Modern Physics 73, 719

Burrows, A., Sudarsky, D., \& Lunine, J. 2003, ApJ 596, 587

Chauvin, G., Lagrange, A.-M., Dumas, C., Zuckerman, B., Mouillet, D., Song, I., Beuzit, J.-L., \& Lowrance, P. 2005, Astronomy and Astrophysics 438, L25

Close, L.M., Lenzen, R., Biller, B.A., Brandner, W., \& Hartung, M. 2005, Science with AO ESO Workshop, 136

Close, L.M., Rainer, R., Guirado, J.C., Nielsen, E.L., Mamajek, E.E., Brandner, W., Hartung, M., Lidman, C., \& Biller, B.A. 2005, Nature, 433, 286

Hartung, M., Herbst, T.M., Close, L.M., Lenzen, R., Brandner, W., Marco, O., \& Lidman, C. 2004, Astronomy and Astrophysics, 421, L17

Kaisler, D., Zuckerman, B., \& Becklin, E. 2003, ASP Conference Series 294, 91

Lenzen, R., Close, L., Brandner, W., Hartung, M., \& Biller, B. 2004, Proc. SPIE 5492

Lenzen, R., Close, L., Brandner, W., Biller, B., \& Hartung, M. 2005, Science with AO ESO Workshop, 46

Lineweaver, C.H. \& Grether, D. 2003, ApJ 598, 1350

Marcy, G., Butler, R., Fischer, D., \& Vogt, S. 2003, ASP Conference Series 294, 1

Marois, C., Doyon, R., Racine, R., \& Nadeau, D. 2000, PASP 112, 91

Marois, C., Doyon, R., Racine, R., \& Nadeau, D. 2002, Proc. SPIE 4008, 788

Marois, C., Doyon, R., Nadeau, D., Racine, R., Riopel, M., Vallée, P., \& Lafrenière, D 2005, PASP, 117, 745

Masciadri, E., Mundt, R., Henning, T., Alvarez, C., \& Barrado y Navascues, D. 2005, ApJ 625, 1004

Masciadri, E. et al. 2006, IAUC 200 proceedings

McCaughrean, M.J., Close, L.M., Scholz, R.-D., Lenzen, R., Biller, B., Brandner, W., Hartung, M., \& Lodieu, N. 2004, Astronomy and Astrophysics, 413, 1029

Mugrauer, M. \& Neuhäuser, R. 2005, MNRAS, 361, L15 
Neuhäuser, R., Guenther, E.W., Wuchterl, G., Mugrauer, M., Bedalov, A., \& Hauschildt, P.H. 2005, Astronomy and Astrophysics 435, L13

Nielsen, E.L., Close, L.M., Guirado, J.C., Biller, B.A., Lenzen, R., Brandner, W., Hartung, M., \& Lidman, C. 2005, to appear in Astronomische Nachrichten

E. Nielsen et al. 2005, this conference.

Racine, R., Walker, G.A.H., Nadeau, D., Doyon, R., \& Marois, C. 1999, Proceedings of the Astronomical Society of the Pacific 111, 587

Schneider, G., Becklin, E., Close, L., Figer, D., Lloyd, J., Macintosh, B., Hines, D., Max, C., Potter, D., Rieke, M., Scoville, N., Thompson, R., Weinberg, A., \& Windhorst, R. 2003, solicited by STScI in preparation for HST Cycle 12

\section{Discussion}

LAGRANGE: In your companion mass vs. AU plot (see Figure 3 in this paper), which planets are cool enough to have $\mathrm{CH}_{4}$ and hence be detectable with the SDI device?

BILler: No planets hotter than $1400 \mathrm{~K}$ are considered detected since their $\mathrm{CH}_{4}$ absorption features are too weak to use the SDI technique (see methane cutoff line at $\mathrm{T}_{\text {eff }}=1400$ $\mathrm{K}$ in Figure 3.) 

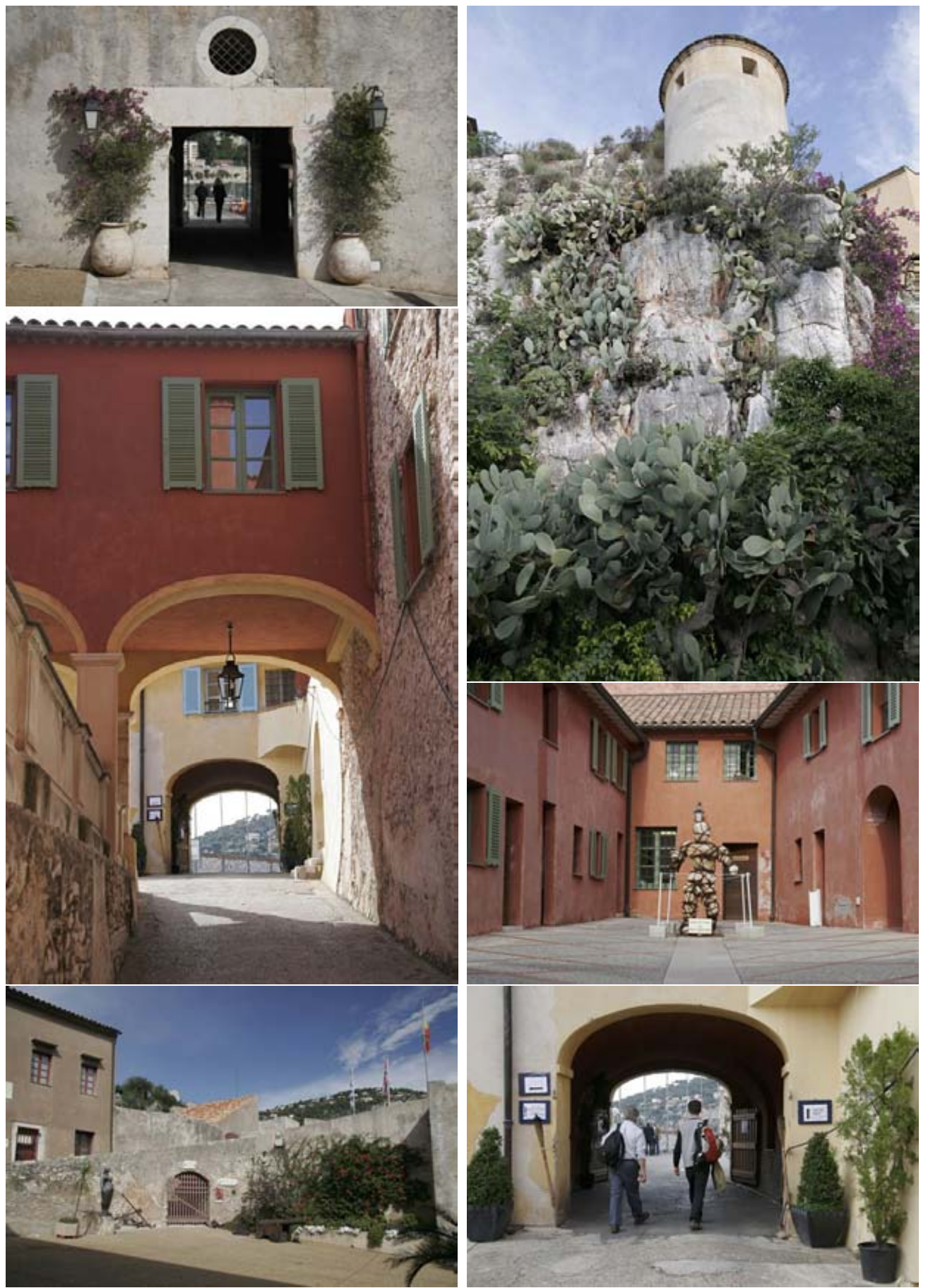

All photographs: Laurent Thareau [1.thareau@free.fr]. 